\title{
EFFECT OF PKa VALUE UPON INTERCALATION OF HETEROCYCLIC COMPOUND INTO LAYERED PHOSPHATE
}

\author{
MAYUMI DANJO, KAZUYO KAKIGUCHI, TOMOMI YANAGIDA, \\ YOSHINOBU BABA, MITSUTOMO TSUHAKO, SHUNRO \\ YAMAGUCHI*, HIROYUKI NARIAI**, AND ITARU MOTOOKA** \\ Kobe Pharmaceutical University; Motoyama-kitamachi, Higashinada-ku, \\ Kobe 658, Japan \\ * The Institute of Scientific and Industrial Research, Osaka University, \\ Mihogaoka, Ibaraki, Osaka 567, Japan \\ ** Department of Chemical Science and Engineering, Faculty of \\ Engineering, Kobe University; Rokkodai-cho, Nada-ku, Kobe 657, Japan
}

\begin{abstract}
The arrangement of heterocyclic compound in the intercalation compounds of the $\alpha$ - and $\gamma$-type zirconium and titanium phosphates, and the effect of pKa values of guest compounds on the intercalation into layered phosphates, were examined. An appreciable difference in the arrangements of aminopyridines between $\alpha$ - and $\gamma$-types was not found. The intercalation of heterocyclic compounds into layered phosphates depended on their pKa values. In the intercalation of heterocyclic compounds, $\alpha$-type layered phosphate was more strongly influenced by the pKa values of the guest compound than that of $\gamma$-type.
\end{abstract}

\section{INTRODUCTION}

Layered phosphates of tetravalent metals are typical ion exchangers with layered structure.1) They are also known as host material which can intercalate various organic bases such as amines. 2) Recently, the intercalation compounds of significant guests such as medicines and bactericides have attracted the extensive attention.3) Because, their intercalation into inorganic layered phosphates may lead to new functional materials that are characterized by controlling the release of guest compound, maximum duration of effectiveness, and good thermal stability. Hence the more detailed informations on the intercalation of aromatic (heterocyclic) compounds into the layered phosphate have been required.

The intercalation of aromatic compounds has been widely investigated, in 
particular the intercalation mechanisms of heterocyclic compounds such as pyridine4), aminopyridine5) and imidazole6), the arrangement of guest compounds in the interlayer region of layered phosphate and thermal stability of the intercalation compounds have been discussed by many investigators. But the discussions described in their papers have been restricted to a few kinds of heterocyclic compounds. In the present research, thus, we investigated the intercalation of a wider range of heterocyclic compounds, and the effect of $\mathrm{pKa}$ values of guest compounds upon the intercalation into layered phosphates.

Zirconium and titanium phosphates were represented by two fundamental forms: $\alpha-\mathrm{Zr}\left(\mathrm{HPO}_{4}\right)_{2} \cdot \mathrm{H}_{2} \mathrm{O}(\alpha-\mathrm{ZrP})^{7)}$ and $\left.\gamma-\mathrm{Zr}\left(\mathrm{H}_{2} \mathrm{PO}_{4}\right)\left(\mathrm{PO}_{4}\right) \cdot 2 \mathrm{H}_{2} \mathrm{O}(\gamma-\mathrm{ZrP}) 8\right)$, and $\alpha-$ $\left.\mathrm{Ti}\left(\mathrm{HPO}_{4}\right)_{2} \cdot \mathrm{H}_{2} \mathrm{O}(\alpha-\mathrm{TiP})^{9}\right)$ and $\left.\gamma-\mathrm{Ti}\left(\mathrm{H}_{2} \mathrm{PO}_{4}\right)\left(\mathrm{PO}_{4}\right) \cdot 2 \mathrm{H}_{2} \mathrm{O}(\gamma-\mathrm{TiP})^{9}\right)$, respectively. The structural difference between $\alpha$-and $\gamma$-types is considerable and predicted to affect the facility of intercalation and the arrangement of guest compounds in the interlayer region of layered phosphates. Hence, the arrangements of aminopyridine in $\alpha$ - and $\gamma$-types were compared, in addition to the effect of $\mathrm{pKa}$ values of guest compounds upon the intercalation into layered phosphates.

\section{EXPERIMENTAL}

All heterocyclic compounds were of reagent grade from Wako Chemical Industries Ltd. and were used without further purification. $\gamma$-ZrP was supplied by Daiichi Kigenso Chemical Industries Ltd. Other layered phosphates were prepared according to the procedures described in the previous papers.10-12)

Layered phosphates $(5.0 \mathrm{~g})$ were suspended in $5 \mathrm{w} / \mathrm{v} \%$ aqueous solution of heterocyclic compounds (listing in table 1) in an Erlenmeyer flask and a suspension was stirred at $70^{\circ} \mathrm{C}$ for $5 \mathrm{~h}$. The resultant product was filtered, washed with distilled water, and dried in air. The X-ray diffraction patterns were measured with a Rigaku Geigerflex 2011 using Ni-filtered $\mathrm{CuK} \alpha$ radiation to monitor all new phases.

\section{RESULTS AND DISCUSSION}

The arrangement of heterocyclic compound in $\gamma$-type layered phosphate was compared with that in $\alpha$-type. The arrangements of aminopyridines in the interlayer region were discussed from an increment in the basal spacing and molecular sizes of aminopyridines. Figure 1 shows the $\mathrm{X}$-ray diffraction patterns 


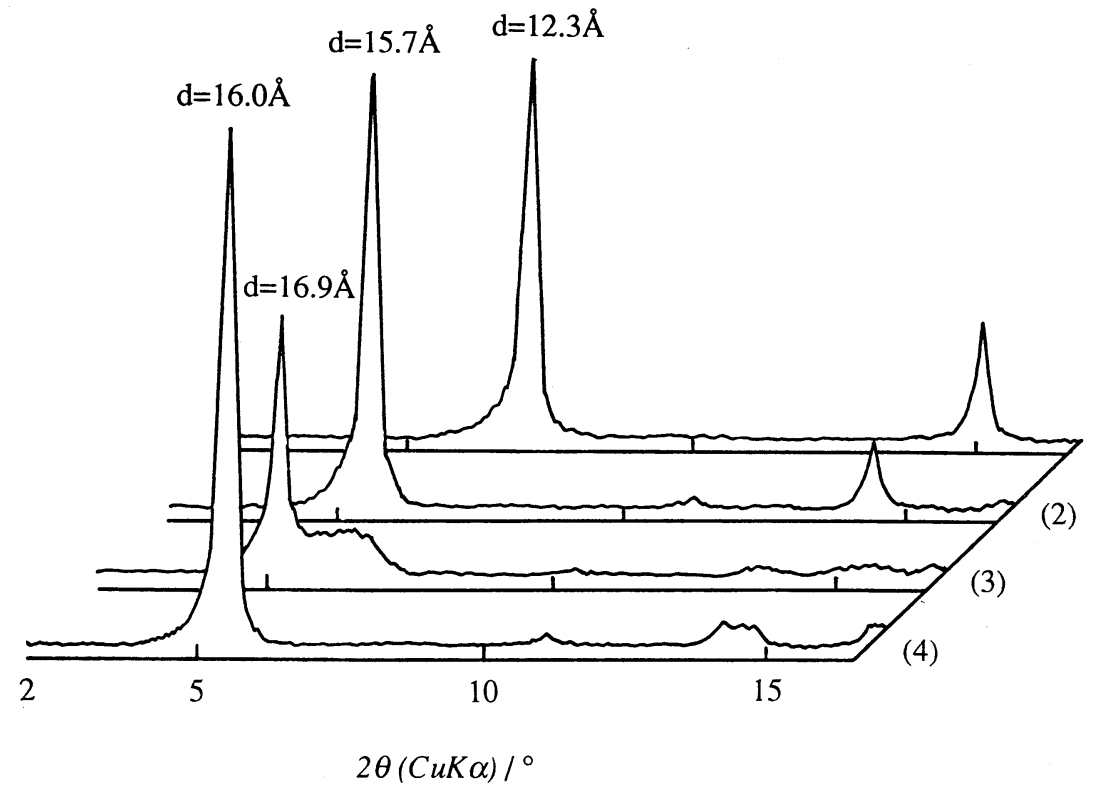

FIGURE 1 X-ray diffraction patterns of aminopyridine intercalation compounds of $\gamma$-ZrP

(1) $\gamma$-ZrP, (2) 2-aminopyridine, (3) 3-aminopyridine, (4) 4-aminopyridine

of the 2-, 3-, and 4-aminopyridine intercalation compounds of $\gamma$-ZrP. As can be seen, new distinct diffraction peaks appeared at $2 \theta=5.6^{\circ}$ (interlayer distance, $\mathrm{d}=15.7 \AA), 5.2^{\circ}(16.9 \AA)$, and $5.5^{\circ}(16.0 \AA)$ in Fig. 1-(2-4), respectively, and broad peaks at $2 \theta=5.9 \sim 6.8^{\circ}$ in Fig. $1-(3)$. The peak at $2 \theta=7.2(d=12.3 \AA)$ of original $\gamma$ $\mathrm{ZrP}$ host phase in Fig. 1-(1) completely disappeared. Such a low degree shift in the diffraction peak represents that aminopyridines are intercalated into the layer of $\gamma-\mathrm{ZrP}$ to expand the interlayer distance.

The interlayer distances of aminopyridine intercalation compounds of other layered phosphates were listed in Table 1. The samples obtained by the reactions of 3-aminopyridine with $\gamma$-layered phosphates were dark brown. The arrangements of aminopyridines in $\alpha-\mathrm{ZrP}$ have been described in the previous paper.5) Since the interlayer distances of the intercalation compounds of $\alpha$-TiP were similar to those of $\alpha-\mathrm{ZrP}$, the arrangement of aminopyridine in two hosts may be identical, except that the peak at $d=17.7 \AA$ observed in the $X$-ray diffraction pattern of 2-aminopyridine intercalation compound of $\alpha-\mathrm{ZrP}$, 
TABLE 1 Interlayer distances of the layered phosphates intercalated various heterocyclic compound

\begin{tabular}{lccccc}
\hline Heterocyclic & $\mathrm{pKa}$ & \multicolumn{5}{c}{ Interlayer distance / } \\
\cline { 3 - 6 } compound & & $\alpha$-ZrP & $\gamma$-ZrP & $\alpha$-TiP & $\gamma$-TiP \\
\hline Pyrazine & 0.7 & - & - & - & - \\
Pyrimidine & 1.3 & 9.8 & - & - & - \\
Pyridazine & 2.3 & 10.9 & - & - & 11.8 \\
Pyrazole & 2.6 & 10.6 & - & - & - \\
Nicotinamide & 3.5 & 10.6 & 17.6 & - & 17.0 \\
Pyridine & 5.4 & 11.0 & - & 11.0 & - \\
3-Aminopyridine & 6.0 & 12.6 & 16.9 & 11.8 & 16.7 \\
& & 11.0 & $13.0 \sim 15.0$ & & 18.4 \\
2-Aminopyridine & 6.8 & 17.7 & 15.7 & 13.0 & 16.1 \\
Imidazole & 13.0 & 16.4 & 15.0 & amor. & 16.1 \\
4-Aminopyridine & 9.2 & 12.8 & 16.0 & 11.9 & 15.5 \\
\hline
\end{tabular}

The symbol "-" shows that heterocyclic compound was not intercalated.

representing the bilayer structure, did not appear in $\alpha$-TiP.

The interlayer distances of the aminopyridine intercalation compounds of $\gamma$-ZrP and $\gamma$-TiP were also similar each other. Since aminopyridine has two active sites (nitrogen atoms in the amino group and the pyridine ring), some arrangements of aminopyridine in the interlayer region can be proposed. In the case of $\alpha-\mathrm{ZrP}$, the phases with two monolayer structures, in which aminopyridine binds to the interlayer $\mathrm{POH}$ sites through the amino group and the nitrogen in the pyridine ring, and a bilayer structure, were found. The new phases with $d=15.5 \sim$ $16.1 \AA$ of $\gamma$-layered phosphates have a monolayer structure, regarded from $\Delta \mathrm{d}=6.3$ $\sim 6.9 \AA$, the $\Delta \mathrm{d}$ value is the increment in the basal spacing calculated by subtracting the thickness of the sheets (anhydrate form: $9.5 \AA$ for $\gamma$-ZrP and $9.2 \AA$ for $\gamma$-TiP13) from the interlayer distances of the intercalation compounds. On the other hand, molecular size of aminopyridine (the distance between an amino 
group or a nitrogen in the pyridine ring and the atom of the most apart) is $4.62 \sim$ 5.58 or $3.92 \sim 4.62 \AA$, respectively. Pyridine was not intercalated into $\gamma$-layered phosphates due to the steric hindrance around nitrogen, therefore, aminopyridine binds to the interlayer $\mathrm{P}(\mathrm{OH})_{2}$ through the amino group.

The appearance of the phase with $\mathrm{d}=18.4 \AA$ in the 2 -aminopyridine intercalation compound of $\gamma$-TiP suggests the formation of a bilayer structure, because $\Delta \mathrm{d}$ value $(9.2 \AA)$ is appreciable larger than a molecular size of 2 aminopyridine. The formation of a bilayer was observed only in 2-aminopyridine intercalation compound of $\gamma$-TiP, in analogy with $\alpha-\mathrm{ZrP}$.

Next, the effect of $\mathrm{pKa}$ of heterocyclic compounds upon their intercalations into layered phosphates was discussed. Table 1 lists heterocyclic compounds used in the present experiment and their $\mathrm{pKa}$ values. Their intercalations into layered phosphates were proved from the $\mathrm{X}$-ray diffraction patterns. The interlayer distances of the new phases are shown in Table 1. As can been seen, the larger the pKa value of heterocyclic compound is, the easier the intercalation tends to take place, for the whole layered phosphates. In particular, the intercalation of heterocyclic compounds into $\alpha$-type layered phosphates greatly depended on the pKa of guest compounds. On the other hand, the irregular cases were present in the intercalation into $\gamma$-type layered phosphates. For example, despite the $\mathrm{pKa}$ value of pyridine is larger than that of nicotinamide, the former can not be intercalated and the latter can into $\gamma-\mathrm{ZrP}$ and $\gamma$-TiP. However, the pKa dependence of the intercalation into layered phosphates is substantially recognized.

Needless to say, it is seen that the factor influencing the intercalation is not only the pKa value of guest compound, but also the structure of guest compound including the molecular size and the bulk around the active site. The structural factors of guest compounds cause the irregularities in the intercalation of heterocyclic compounds into $\gamma$-ZrP and $\gamma$-TiP. On the other hand, in $\alpha$-layered phosphate every phosphorus atom has an acid group (POH). In contrast, in the $\gamma$ layered phosphate structure there are two different types of phosphorus (orthophosphate $\mathrm{PO}_{4}{ }^{3-}$ and dihydrogen phosphate $\mathrm{H}_{2} \mathrm{PO}_{4}^{-}$). The acid center of $\gamma-$ layered phosphate is placed only on the dihydrogen phosphate group. It is also known that the free area around the active site of $\gamma$-layered phosphate is smaller than that of $\alpha$-phosphate, due to the more dense structure of the $\gamma$-phosphate, in 
comparison with the $\alpha$-phosphate. Hence, the intercalation of heterocyclic compound into $\gamma$-layered phosphate may be more greatly influenced by the structure of guest compound than that into $\alpha$-phosphate.

In addition, the difference between metals constituting layered phosphate was proved. The minimum pKa value of heterocyclic compound, permitting the intercalation into $\alpha-\mathrm{ZrP}$ and $\alpha$-TiP, is about 1 and 4 5, respectively. Because the structure of $\alpha$-TiP is isostructural with $\alpha-\mathrm{ZrP}, 9)$ it is difficult to explain the reason of these results from a structural point of view. However, such differences represent that nature of metal constituting the layered structure influence the character of the layered phosphate.

The intercalation into layered phosphates is greatly controlled by the $\mathrm{pKa}$ values of the guest molecules and the structures of host compounds. Thus, in order to make a success of the intercalation, the pKa values of guest compounds and the character of the layered phosphate should be sufficiently taken into account.

\section{REFERENCES}

1 A. Clearfield, "Inorganic Ion Exchange Materials," edited by A. Clearfield, CRC Press, Inc., Boca Raton, Florida (1982), p. 1.

2 U. Costantino, "Inorganic Ion Exchange Materials," edited by A. Clearfield, CRC Press, Inc., Boca Raton, Florida (1982), p. 111.

3 M. Danjo, Y. Baba, M. Tsuhako, S. Yamaguchi, H, Nariai, and I. Motooka, Bull. Chem. Soc. Jpn., 68, 1607(1995).

4 S. Yamanaka, Y. Horibe, and M. Tanaka, J. Inorg. Nucl. Chem., 38, 323 (1976).

5 M. Danjo, Y. Baba, M. Tsuhako, H. Nariai, and I. Motooka, Phosphorus Research Bulletin, 3, 25(1993).

6 U. Costantino, M. A. Massucci, A. Ginestra, A. M. Tarola, and L. Zampa, J. Incl. Phen., 4, 147(1986).

7 J. M. Troup, A. Clearfield, Inorg. Chem., 16, 3311(1977).

8 D. M. Poojary, B. Shpeizer, and A. Clearfield, J. Chem. Soc. Dalton Trans., 1995, 111.

9 A. N. Christensen, E. K. Andersen, I. G. K. Andersen, G. Alberti, M. Nielsen, and M. S. Lehmann, Acta Chem. Scand., 44, 865(1990).

10 M. Tsuhako, Y. Horii, K Kawamoto, N. Kawataka, H. Nariai, and I. Motooka, Nippon Kagaku Kaishi, 1988, 1810.

11 M. Tsuhako, Y. Horii, H. Nariai, and I. Motooka, Nippon Kagaku Kaishi,. 1987, 1541.

12 M. Tsuhako, K. Kawamoto, Y. Horii, H. Nariai, and I. Motooka, Nippon Kagaku Kaishi,. 1990, 740.

13 U. Costantino, J. Inorg. Nucl. Chem., 43, 1895 (1981). 